\title{
O AGENTE DE CONHECIMENTO PERSPECTIVAS NO ÂMBITO DA TRADUÇÃO DO CONHECIMENTO NA SAÚDE PÚBLICA
}

\author{
Juliana Gonçalves Reis \\ Fundação Oswaldo Cruz, Escola Politécnica de Saúde Joaquim Venâncio \\ julianareis20@gmail.com
}

\begin{abstract}
Resumo
Este artigo apresenta o Knowledge Broker como um agente de conhecimento para intermediar informação e comunicação bidirecionada a pesquisadores e às partes interessadas em utilizarem os resultados de pesquisa, com finalidade de facilitar a tradução de conhecimento na saúde pública. Destaca a ausência deste perfil no Brasil e propõe uma agenda de trabalho focada em 1- Delineamento de ecossistemas de conhecimento para garantir a especificidade de interlocução com as partes interessadas; 2- Estratégias de fortalecimento de redes de colaboração; 3 - Boas práticas de mobilização de conhecimento com a finalidade de desenvolver, aplicar e avaliar estratégias e métodos para que o conhecimento faça diferença positiva e 4 - Desenvolvimento de competência para o impacto da pesquisa com objetivo de tornar os resultados de pesquisa e avaliação de dados úteis para a vida das pessoas e da sociedade.
\end{abstract}

Palavras-chave: Tradução do conhecimento. Agente do conhecimento. Saúde Pública.

\section{THE KNOWLEDGE AGENT PERSPECTIVES IN THE CONTEXT OF THE TRANSLATION OF KNOWLEDGE IN PUBLIC HEALTH}

\begin{abstract}
This article introduces Knowledge Broker as a knowledge agent for brokering information and two-way communication to researchers and stakeholders interested in using search results. In order to facilitate the translation of knowledge in public health. Highlights the absence of this profile in Brazil and proposes a work agenda focused on 1- Design of knowledge ecosystems to ensure the specificity of dialogue with stakeholders; 2 Strategies for strengthening collaborative networks; 3 - Good knowledge mobilization practices for the purpose of developing, applying and evaluating strategies and methods for knowledge to make a positive difference; and 4 Developing competence for research impact with a view to making research results and data evaluation. useful for the lives of people and society.
\end{abstract}

Keywords: Knowledge translation. Knowledge broker. Public health. 


\section{INTRODUÇÃO}

A tradução do conhecimento é definida como um processo dinâmico e interativo que inclui síntese, disseminação, troca e aplicação do conhecimento de forma ética para melhorar a saúde, fornecer serviços e produtos de saúde mais eficazes e fortalecer o sistema de saúde, conforme estabelece o Canadian Institutes of Health Research (CIHR), um dos principais fomentadores da tradução do conhecimento como política pública, tornando o conhecimento para ação uma estratégia nacional.

Estudos de McLean(2012) e Graham(2018) buscaram propor um protocolo de estudo para os envolvidos na teoria, prática e avaliação de tradução de conhecimento. Além disso, Gagliardi (2016) sinalizou a importância de construir uma agenda de pesquisa para a tradução integrada do conhecimento, pois estratégias unidirecionais de sínteses e resumos de pesquisa apresentavam impacto inconsistente no uso real da pesquisa e na melhoria da prestação de cuidados. Ainda esses autores, destacaram a necessidade de mais pesquisas para estabelecer uma terminologia comum, processos efetivos para formação e colaboração de parcerias. Bem como papéis dos participantes para possibilitar condições das intervenções e medidas de impacto.

\section{A INICIATIVA K* PARA UNIFICAR OS TERMOS}

Diversos termos como gestão do conhecimento, transferência de conhecimento, mobilização do conhecimento, tradução integrada do conhecimento entre outros têm ampliado este campo e em 2012, a iniciativa chamada $K^{*}$ chegou ao documento conceitual elaborado por um grupo de especialistas reunidos em Ontário, Canadá. A finalidade foi de alcançar a compreensão mútua e reduzir a fragmentação dos termos do campo de conhecimento, a fim de evitar a reinvenção da roda.

Este consenso, ofereceu uma linguagem comum e a definição dos princípios compartilhados por todas as funções e processos do componente $\mathrm{K}^{*}$. Neste documento é utilizado o termo Knowledge brokering para troca de conhecimento bidirecional sobre uma questão, que promove aprendizado e geralmente envolvem um Knowledge Broker ou um intermediário. 


\section{AGENTE DO CONHECIMENTO INTERMEDIADOR DE INFORMAÇÃO E COMUNICAÇÃO}

O Knowledge Broker (BK) é um termo em inglês referente a uma estratégia emergente no Canadá no final dos anos 90.Tornou-se popular na tradução de conhecimento com objetivo de promover a interação do conhecimento entre pesquisadores e usuários finais.Atualmente, representa um perfil profissional abrangente fundamental para facilitar a informação e a comunicação, melhorar o compartilhamento de conhecimento entre as partes interessadas, apoiar o aprendizado, capacitar para localizar, avaliar e traduzir evidências no contexto local e mobilizar conhecimento para a ação.

Neste texto utilizarei para o termo Knowledge Broker, a versão da língua portuguesa agente de conhecimento, considerando um artigo de Rezende (2002), que faz menção ao termo e destaca a presença de novos agentes de conhecimento, dentre eles, os agentes gestores do conhecimento, como uma nova categoria de profissionais, cujo papel é a administrar o capital intelectual da organização.

A perspectiva da ascensão do agente de conhecimento não é nova. Nos setores comerciais e agrícola tem evidências consideráveis da avaliação do impacto dessa estratégia. No setor da saúde, nos últimos anos o interesse pela intermediação do conhecimento tem crescido. Na revisão sistemática de Bornbaum (2015), exploraram as funções predominantes e a eficácia de agentes de conhecimento em ambientes de cuidados de saúde, confirmaram que os papéis desempenhados se enquadram em três domínios abrangentes: gerentes de conhecimento, agentes de vinculação e capacitadores. Entretanto, os autores apontam a necessidade de novas pesquisas para avaliar o impacto potencial dos agentes de conhecimento na educação, na prática e na política.

O artigo de Traynor, DeCorby e Dobbins (2014), descreveu um estudo de método misto que agentes de conhecimento em saúde pública aumentaram a capacidade individual, melhorando o conhecimento e a habilidade em buscar, avaliar criticamente e aplicar evidências de pesquisa a questões baseadas na prática. A capacidade organizacional foi aprimorada com forte apoio em políticas e de gestão da pesquisa.

Anteriormente, em um outro artigo Dobbins (2009) descreveu a implementação da função do agente de conhecimento entre os departamentos de saúde pública no Canadá, como parte de um estudo controlado randomizado de avaliação de três estratégias de tradução de conhecimento. A intervenção do agente de conhecimento foi intermediação de conhecimento e 
tarefas específicas que incluíam assegurar que evidências relevantes de pesquisas fossem transferidas para os tomadores de decisão de saúde pública.

Nesse sentido, as atividades de base de conhecimento foram classificadas em avaliação individual e organizacional, explorando o horizonte, gestão de conhecimento, tradução e troca de conhecimento, desenvolvimento, manutenção e facilitação de redes. Essas classes foram resultados das pesquisas conduzidas por Dobbins na Universidade McMaster, sobre o papel do agente de conhecimento em serviços de saúde pública, que propõem um método que poderia ser usado para informar o desenvolvimento e a implementação de uma intervenção do agente de conhecimento em contextos de saúde pública.

Em um outro estudo Kim (2018), o agente de conhecimento é parte integrante para compor a tríade central de interação, juntamente com os resultados de pesquisa e os seus usos. Essa tríade, configura a estrutura de utilização de pesquisa para informar políticas e programas globais de saúde e desenvolvimento.

Glegg e Hoens (2016) elaboraram um modelo de domínios do papel do agente de conhecimento para o ambiente de saúde. Neste modelo, as funções de intermediação de conhecimento abrangem o gerenciamento da informação, o agente de vinculação, o construtor de capacidade, o facilitador e o avaliador.

\section{CONSIDERAÇÕES FINAIS}

No Brasil, de modo específico, não se tem debatido o perfil de um agente de conhecimento a ser definido para que o mesmo possa intermediar a tradução do conhecimento na saúde pública. Por outro lado, no cenário internacional acredita-se que a pesquisa intermediada por um agente do conhecimento com as partes interessadas e pesquisadores, utilizando-se como ponto de partida a informação e a comunicação bidirecional, são capazes de ampliar a capacidade de formação e fortalecimento de redes para melhoria na qualidade do serviço de prestação de cuidados.

Nessa perspectiva, propõe-se como agenda inicial para construção desse perfil nos espaços de pesquisa no Brasil: 1- Delineamento de ecossistemas de conhecimento para garantir a especificidade de interlocução com as partes interessadas; 2- Estratégias de fortalecimento de redes de colaboração; 3 - Boas práticas de mobilização de conhecimento com a finalidade de desenvolver, aplicar e avaliar estratégias e métodos para que o conhecimento faça diferença positiva e 4 - Desenvolvimento de competência para o impacto da pesquisa com objetivo de 
- INOVAção

tornar os resultados de pesquisa e avaliação de dados úteis para a vida das pessoas e da sociedade. 


\section{REFERENCIAS}

CANADIAN INSTITUTES OF HEALTH RESEARCH (CIHR) Knowledge Translation Definition. Disponível em: http://www.cihr-irsc.gc.ca/e/29418.html\#2

DOBBINS M, ROBESON P, Ciliska D, HANNA S, CAMERON R, O'Mara L, et al. A description of a knowledge broker role implemented as part of a randomized controlled trial evaluating three knowledge translation strategies. Implementation Science [Internet]. dezembro de 2009 [citado 21 de abril de 2018];4(1). Disponível em: http://implementationscience.biomedcentral.com/articles/10.1186/1748-5908-4-23

GAGLIARDI AR, KOTHARI A, GRAHAM ID. Research agenda for integrated knowledge translation (IKT) in healthcare: what we know and do not yet know. Journal of Epidemiology and Community Health. fevereiro de 2017;71(2):105-6. Disponível em: http://jech.bmj.com/content/early/2016/09/19/jech-2016-207743

GLEGG SM, HOENS A. ROLE Domains of Knowledge Brokering: A Model for the Health Care Setting. Journal of Neurologic Physical Therapy. abril de 2016;40(2):115-23. Disponível em:

https://journals.lww.com/jnpt/fulltext/2016/04000/Role_Domains_of_Knowledge_Brokering_ __A_Model_for.7.aspx

KIM C, WILCHER R, PETRUNEY T, KRUEGER K, WYNNE L, Zan T. A research utilisation framework for informing global health and development policies and programmes. Health Research Policy and Systems [Internet]. dezembro de 2018 [citado 21 de abril de 2018];16(1). Disponível em: https://health-policysystems.biomedcentral.com/articles/10.1186/s12961-018-0284-2

MCLEAN RKD, GRAHAM ID, BOSOMPRA K, CHOUDHRY Y, COEN SE, MACLEOD $\mathrm{M}$, et al. Understanding the performance and impact of public knowledge translation funding interventions: Protocol for an evaluation of Canadian Institutes of Health Research knowledge translation funding programs. Implementation Science [Internet]. dezembro de 2012 [citado 21 de abril de 2018];7(1). Disponível

em: http://implementationscience.biomedcentral.com/articles/10.1186/1748-5908-7-57.

On behalf of the Integrated Knowledge Translation Research Network Project Leads, Graham ID, Kothari A, McCutcheon C. Moving knowledge into action for more effective practice, programmes and policy: protocol for a research programme on integrated knowledge translation. Implementation Science [Internet]. dezembro de 2018 [citado 21 de abril de 2018];13(1). Disponível

em: https://implementationscience.biomedcentral.com/articles/10.1186/s13012-017-0700-y

REZENDE Y. Informação para negócios: os novos agentes do conhecimento e a gestão do capital intelectual. Ci Inf. 2002;31(2):120-8. Disponível em:

http://www.scielo.br/pdf/ci/v31n2/12915.pdf 
SHAXSON L, BIELAK A, AHMED I, BRIEN D, CONANT B, FISHER C, et al. Expanding our understanding of $\mathrm{K}^{*}(\mathrm{KT}, \mathrm{KE}, \mathrm{KTT}, \mathrm{KMb}, \mathrm{KB}, \mathrm{KM}$, etc.) A concept paper emerging from the $\mathrm{K}^{*}$ conference held in Hamilton [Internet]. Ontario, Canadá: United Nations University, Institute for Water, Environment and Health (UNU-INWEH); 2012 p. 88. Disponível em: http://inweh.unu.edu/wpcontent/uploads/2013/05/KStar_ConceptPaper_FINAL_Oct29_WEB.pdf

TRAYNOR R, DECORBY K, DOBBINS M. Knowledge brokering in public health: a tale of two studies. Public Health. junho de 2014;128(6):533-44. Disponível em: http://www.publichealthjrnl.com/article/S0033-3506(14)00032-8/fulltext 